\title{
RNA sequencing profiles and diagnostic signatures linked with response to ramucirumab in gastric cancer
}

\author{
Maxim Sorokin, ${ }^{1,2,3}$ Elena Poddubskaya, ${ }^{1}$ Madina Baranova, ${ }^{4,5}$ Alex Glusker, ${ }^{1}$ \\ Lali Kogoniya, ${ }^{6}$ Ekaterina Markarova, ${ }^{6}$ Daria Allina, ${ }^{1}$ Maria Suntsova, ${ }^{3}$ \\ Victor Tkachev, ${ }^{2}$ Andrew Garazha, ${ }^{2}$ Marina Sekacheva, ${ }^{1}$ and Anton Buzdin ${ }^{1,2,3,7}$ \\ ${ }^{1}$ I.M. Sechenov First Moscow State Medical University, Moscow, 119991, Russia; ${ }^{2}$ Omicsway Corp., Walnut, \\ California 91789, USA; ${ }^{3}$ Shemyakin-Ovchinnikov Institute of Bioorganic Chemistry, Moscow, 117997, Russia; \\ ${ }^{4}$ N.N. Blokhin Russian Cancer Research Center, Moscow, 115478, Russia; ${ }^{5}$ Clinical Center Vitamed, Moscow, \\ 121309, Russia; ${ }^{6}$ M.F. Vladimirsky Moscow Regional Research Clinical Institute, Moscow, 129110, Russia; \\ ${ }^{7}$ Moscow Institute of Physics and Technology, Moscow Region, 141701, Russia
}

Corresponding author: buzdin@oncobox.com

(C) 2020 Sorokin et al. This article is distributed under the terms of the Creative Commons Attribution-NonCommercial

License, which permits reuse and redistribution, except for commercial purposes, provided that the original author and source are credited.

Ontology term: stomach cancer

Published by Cold Spring Harbor Laboratory Press

doi:10.1101/mcs.a004945
Abstract Gastric cancer (GC) is the fifth-ranked cancer type by associated mortality. The proportion of early diagnosis is low, and most patients are diagnosed at the advanced stages. First-line therapy standardly includes fluoropyrimidines and platinum compounds with trastuzumab for HER2-positive cases. For recurrent disease, there are several alternative options including ramucirumab, a monoclonal therapeutic antibody that inhibits VEGF-mediated tumor angiogenesis by binding with VEGFR2, alone or in combination with other cancer drugs. However, overall response rate following ramucirumab or its combinations is $30 \%-80 \%$ of the patients, suggesting that personalization of drug prescription is needed to increase efficacy of treatment. We report here original tumor RNA sequencing profiles for 15 advanced GC patients linked with data on clinical response to ramucirumab or its combinations. Three genes showed differential expression in the tumors for responders versus nonresponders: CHRM3, LRFN1, and TEX15. Of them, CHRM3 was up-regulated in the responders. Using the bioinformatic platform Oncobox we simulated ramucirumab efficiency and compared output model results with actual tumor response data. An agreement was observed between predicted and real clinical outcomes ( $A U C \geq 0.7$ ). These results suggest that RNA sequencing may be used to personalize the prescription of ramucirumab for $\mathrm{GC}$ and indicate potential molecular mechanisms underlying ramucirumab resistance. The RNA sequencing profiles obtained here are fully compatible with the previously published Oncobox Atlas of Normal Tissue Expression (ANTE) data.

[Supplemental material is available for this article.]

\section{INTRODUCTION}

Approximately 1 million patients are annually diagnosed with gastric cancer (GC), and nearly 740,000 die of this disease (Ferlay et al. 2010). Despite recent advances in GC treatment, long-term survival remains poor. Standard first-line therapy for unresectable, advanced, or metastatic gastric adenocarcinoma usually includes fluoropyrimidines and platinum compounds (Al-Batran et al. 2008), whereas more options are available at recurrence: 
ramucirumab (Fuchs et al. 2014), paclitaxel (Ajani et al. 1994), their combination (Wilke et al. 2014), docetaxel (Albertsson et al. 2007), and irinotecan (Thuss-Patience et al. 2011). Moreover, anti-PD1 immunotherapeutics can be considered an emerging treatment modality for metastatic GC (Brar and Shah 2019). In addition, molecular testing is required to define HER2 status of individual GC cases that guides prescription of trastuzumab (Bang et al. 2010). However, selection of other drugs including ramucirumab is not based on any molecular diagnostic features.

Ramucirumab is a monoclonal therapeutic antibody that inhibits VEGF-mediated tumor angiogenesis by binding with VEGFR2 (Krupitskaya and Wakelee 2009). For the recurrent/ advanced/metastatic GC it may be used either as monotherapy (Smit et al. 2018; Yamaguchi et al. 2018) or in combinations with other drugs (e.g., paclitaxel) (Wilke et al. 2014) and therapeutic regimens like FOLFIRI (folinic acid + irinotecan + 5-fluorouracil) or FOLFOX (oxaliplatin + leucovorin + 5-fluorouracil) (Yoon et al. 2016) or cisplatin + capecitabine (Fuchs et al. 2019). In published clinical trials, overall response rate following treatment with ramucirumab or its above combinations was $30 \%-80 \%$ of the patients (Wilke et al. 2014; Yoon et al. 2016; Yamaguchi et al. 2018; Fuchs et al. 2019). The overall response rate includes a proportion of the patients who showed complete or partial response to the treatment or disease stabilization. Moreover, for the outcomes of combinational therapies, the impact of ramucirumab itself was unclear, thus suggesting that personalization of drug prescription is needed to increase efficacy of treatment (Yamaguchi et al. 2018).

Gene expression profiles can be considered attractive next-generation biomarkers for cancer molecular diagnostics (Buzdin et al. 2019b). Expressions of single genes (Patel and Kurzrock 2015) and their statistical combinations (van de Vijver et al. 2002) or activation levels of the whole molecular pathways (Buzdin et al. 2018) may be useful to predict individual tumor response to the therapies. In turn, RNA sequencing is currently accepted as the gold standard analytic approach for high-throughput screening of gene expression (SEQC/ MAQC-III Consortium 2014). Comparison of tumor gene expression profiles with the normal tissues is crucial for understanding individual mechanisms of cancer development, progression, and response to the targeted therapies (Buzdin et al. 2019c; Rodon et al. 2019).

However, RNA sequencing data sets obtained using different equipment, reagents, and protocols may be poorly compatible with each other (Buzdin et al. 2014; Borisov et al. 2019), and ideally the same experimental platform should be used to compare the results (Borisov et al. 2019). We recently published an annotated database of RNA sequencing profiles termed Oncobox Atlas of Normal Tissue Expression (ANTE) (Suntsova et al. 2019) that represents 142 solid tissue samples from human healthy donors killed in road accidents and 17 blood samples from healthy volunteers. It has statistically significant reference groups for 20 human tissues/organs including the stomach.

In this study, we report clinically annotated RNA sequencing profiles for 15 advanced GC patients that are fully compatible with the ANTE collection of normal tissues because they were profiled using the same equipment, reagents, and protocols with the same analytic thresholds. Among the others, the molecular data are linked with the information on clinical response to ramucirumab or its combinations. We used bioinformatic platform Oncobox (Sorokin et al. 2018; Buzdin et al. 2019a; Poddubskaya et al. 2019a) to model ramucirumab efficiency based on RNA sequencing data and compared the output results with the known tumor response information. The Oncobox algorithm builds a personalized rating of target drugs for individual cancer patients. It is based on a simultaneous analysis of gene expression and molecular pathway activation in the patient's tumor and was shown to be effective in a prospective cohort of advanced cancer cases (Poddubskaya et al. 2018, 2019b) and in an ongoing prospective clinical investigation (Poddubskaya et al. 2019a). We observed here a statistically significant coincidence of the predicted and real GC clinical outcomes $(A \cup C \geq 0.7)$. These results demonstrate that gene expression analyses may uncover 
COLD SPRING HARBOR Molecular Case Studies
Ramucirumab response prediction in gastric cancer

molecular mechanisms underlying ramucirumab resistance and, more importantly, may be useful to personalize prescription of ramucirumab to recurrent GC patients.

\section{RESULTS}

In this study, we investigated links between experimental gene expression profiles established by RNA sequencing of GC biosamples and the patient's clinical responses to the therapy containing therapeutic monoclonal antibody ramucirumab.

\section{Clinical Data}

In this study, we enrolled 15 patients with advanced or metastatic GC. The patients were receiving ramucirumab-containing therapies in two oncology hospitals: M.F. Vladimirsky Moscow Regional Research Clinical Institute and N.N. Blokhin National Medical Research Center of Oncology. In total, there were five female and 10 male patients enrolled with mean ages of 47.2 and $57.6 \mathrm{yr}$ old, respectively (Table 1). All patients had progressive disease before receiving ramucirumab-containing therapy. Ramucirumab was prescribed as second-line therapy for both metastatic and primary tumors according to the National Comprehensive Cancer Network (NCCN) guidelines for stomach cancer (https://www.nccn .org/patients/guidelines/content/PDF/stomach.pdf). In 14 cases, primary GC specimens were analyzed, whereas in one case, a peritoneal metastasis of GC was investigated. The GC histological subtypes were tubular adenocarcinoma $(n=8)$, poorly cohesive adenocarcinoma, signet-ring cell type $(n=5)$, poorly cohesive adenocarcinoma, not otherwise specified (NOS) $(n=1)$, and omental metastasis of mucinous gastric adenocarcinoma $(n=1)$ (Table 2; Fig. 1). PCA analysis indicated that normal stomach and brain tissues from the ANTE database (Suntsova et al. 2019) formed tight clusters, which were not mixed with GC samples from this study (Fig. 1E). In addition, GC samples were closer to normal stomach than to

Table 1. Patients' demographic and clinical information

\begin{tabular}{lllllc}
\hline Patient ID & Sex & Age & Primary tumor/metastasis & TNM & $\begin{array}{c}\text { Number of lines of therapy } \\
\text { prior to ramucirumab }\end{array}$ \\
\hline GC_4 & Male & 67 & Primary & T4aN3M0 & 1 \\
GC_5 & Male & 45 & Primary & T4N1M1 & 4 \\
GC_6 & Male & 65 & Primary & T4bNxM1 & 1 \\
GC_7 & Male & 79 & Primary & T3N0M0 & 2 \\
GC_8 & Female & 51 & n/a & T3NxM1 & 2 \\
GC_9 & Male & 59 & Primary & T4aN3bM0 & 2 \\
GC_11 & Female & 40 & Primary & TxNxM1 & 1 \\
GC_12 & Male & 41 & Primary & T3NxM0 & 1 \\
GC_13 & Female & 54 & Primary & T4aNxM1 & 1 \\
GC_14 & Male & 33 & Primary & T4N1M1 & 3 \\
GC_15 & Female & 31 & Primary & T4N2M1 & 1 \\
GC_16 & Male & 57 & Primary & T4N2M0 & 1 \\
GC_17 & Female & 60 & Peritoneum & T4bN2M1 & 1 \\
GC_18 & Male & 63 & metastasis & & 1 \\
GC_19 & Male & 67 & Primary & T4bN3M1 & 1 \\
\hline
\end{tabular}




\begin{tabular}{ll}
\hline Table 2. Histological typing of gastric tumor samples investigated \\
\hline Patient ID & \multicolumn{1}{c}{ Histology } \\
\hline GC_4 & Poorly cohesive adenocarcinoma, signet-ring cell type \\
GC_5 & Tubular adenocarcinoma \\
GC_6 & Poorly differentiated tubular adenocarcinoma \\
GC_7 & Tubular adenocarcinoma \\
GC_8 & Poorly cohesive adenocarcinoma, signet-ring cell type \\
GC_9 & Poorly differentiated tubular adenocarcinoma \\
GC_11 & Poorly cohesive adenocarcinoma, signet-ring cell type \\
GC_12 & Poorly differentiated tubular adenocarcinoma \\
GC_13 & Poorly cohesive adenocarcinoma, signet-ring cell type \\
GC_14 & Tubular adenocarcinoma \\
GC_15 & Poorly cohesive adenocarcinoma, signet-ring cell type \\
GC_16 & Poorly cohesive adenocarcinoma NOS \\
GC_17 & Omental metastasis of mucinous gastric adenocarcinoma \\
GC_18 & Tubular adenocarcinoma \\
GC_19 & Poorly differentiated tubular adenocarcinoma \\
\hline
\end{tabular}

normal brain, indicating compatibility of the data sets (Fig. 1E). We used normal brain tissues as an outgroup in order to test the hypothesis that cancer and normal data sets in this study are compatible. Compatibility of data sets implies that biological differences (that manifest themselves as variance in gene expression space) are higher than any of the between data set differences. A PCA plot (Fig. 1E) shows that biological differences between gastric and neural tissues are higher than any of differences between two data sets. All patients underwent ramucirumab therapy either as monotherapy $(n=7)$ or in combination with paclitaxel $(n=6)$ or FOLFIRI regimen $(n=2)$ (Table 3$)$. The registered clinical outcomes of treatment were "partial response," "stable disease," and "progressive disease." In this study, the patients were classified as either responders_for "partial response" and "stable disease" outcomes_or nonresponders-for "progressive disease" outcomes (Table 3).

\section{RNA Sequencing and Molecular Data Quality Control}

The GC tissue specimens were the formalin-fixed paraffin-embedded (FFPE) tissue blocks stored in a clinical diagnostic laboratory for 4-49 mo before extraction of RNA. Previously we used the same experimental protocol for generating the Oncobox atlas of RNA sequencing profiles for normal human tissues from healthy donors (Suntsova et al. 2019). We found that the profiles with the number of sequencing reads mapped on human genes exceeding 2.5 million were clustering tissue-specifically, whereas for the profiles with a lower number of mapped reads we observed biased clustering. A quality control (QC) threshold of 2.5 million gene-mapped reads was, therefore, established to identify highquality RNA sequencing profiles (Suntsova et al. 2019). In this study, we used the same Oncobox RNA sequencing and data processing protocol. All the current 15 GC RNA sequencing profiles passed the $\mathrm{OC}$ threshold and were further investigated. The original sequencing data were deposited to the National Center for Biotechnology Information (NCBI) Sequence Read Archive (SRA) with accession number PRJNA562149. Summary statistics of RNA sequencing (number of unmapped, uniquely mapped, and multimapped reads as well as average exon coverage) are depicted in Supplemental Table S1. 
A

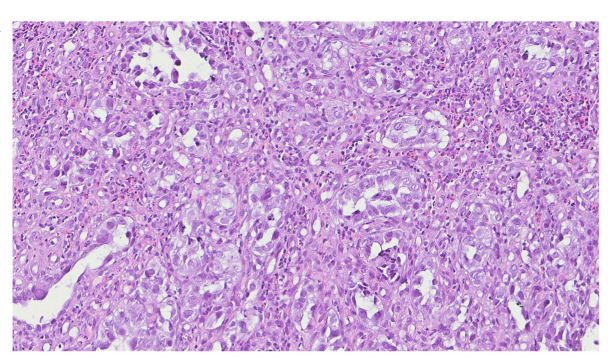

C

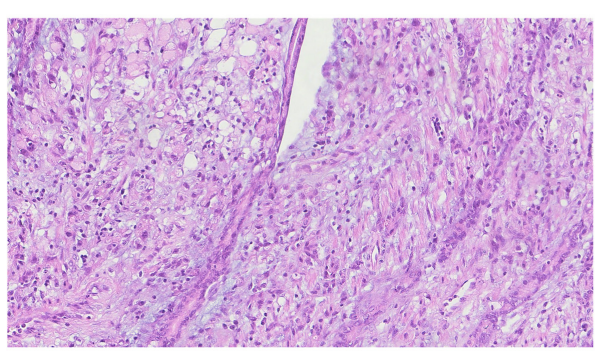

E

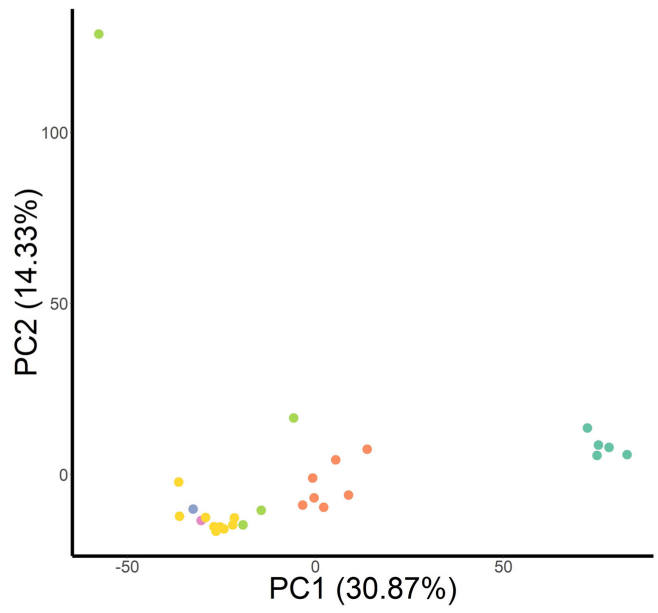

B

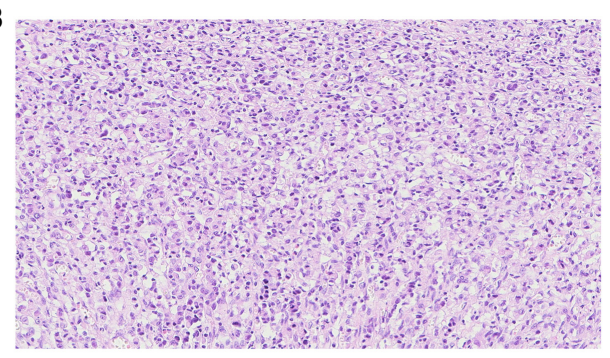

D

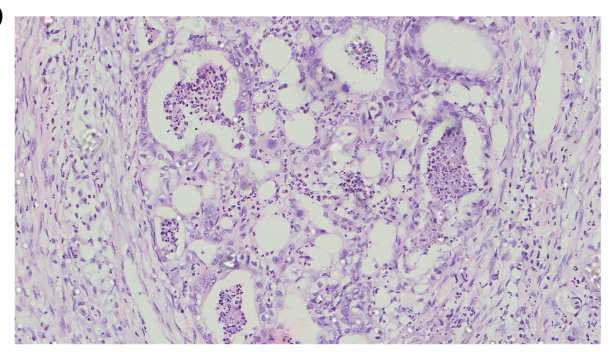

- Normal Brain

- Normal Stomach

- Omental metastasis of mucinous gastric adenocarcinoma

- Poorly cohesive adenocarcinoma NOS

- Poorly cohesive adenocarcinoma, signet-ring cell type

- Tubular adenocarcinoma

Figure 1. Histological subtypes of gastric cancer (GC) samples and principal component analysis (PCA). (A) Tubular adenocarcinoma; (B) poorly cohesive adenocarcinoma, signet-ring cell type; (C) poorly cohesive adenocarcinoma, NOS; (D) omental metastasis of mucinous gastric adenocarcinoma; and (E) PCA of stomach cancer samples, normal stomach samples from the ANTE database, and normal brain samples (outgroup) from the ANTE database (Suntsova et al. 2019).

\section{Differentially Regulated Genes and Molecular Pathways}

According to differentially expressed gene analysis using DESeq2 software, only three genes passed the adjusted $P$-value threshold of 0.05 for "responders" on ramucirumab-containing therapies versus "nonresponders." These genes were CHRM3 (cholinergic receptor muscarinic 3), LRFN1 (leucine rich repeat and fibronectin type III domain containing 1), and TEX15 (testis expressed 15, meiosis and synapsis associated). Of them, CHRM3 was up-regulated in the responders, whereas the other genes were down-regulated. We found no previous literature reports on the implication of these genes in GC. However, CHRM3 is involved in upregulation of MAPK signaling and invasion and migration of prostate and colorectal cancer cells (Belo et al. 2011; Zheng et al. 2019), and its genetic variants are associated with polycystic ovary syndrome (Kim et al. 2019) and bladder cancer (Wang et al. 2016) in Korean and 
Table 3. Ramucirumab treatment regimen for patients enrolled and their clinical outcome

\begin{tabular}{|c|c|c|c|}
\hline Patient ID & Treatment regimen & Clinical outcome & PFS (months) \\
\hline GC_4 & $\begin{array}{l}\text { Paclitaxel }\left(80 \mathrm{mg} / \mathrm{m}^{2} \text { days } 1,8,15\right)+\text { ramucirumab }(8 \mathrm{mg} / \mathrm{kg} \text { days } 1,15)-28 \mathrm{~d} \\
\text { cycles, followed by } 12 \text { cycles of ramucirumab monotherapy } \\
\text { (ramucirumab } 8 \mathrm{mg} / \mathrm{kg} \text { days } 1,15)-28 \mathrm{~d} \text { cycles }\end{array}$ & Stable disease & 11 \\
\hline GC_5 & Ramucirumab monotherapy & Progressive disease & 1 \\
\hline GC_6 & $\begin{array}{l}\text { Seven courses: FOLFIRI (fluorouracil + leucovorin + irinotecan) + ramucirumab } \\
(8 \mathrm{mg} / \mathrm{kg} \text { days } 1,15)\end{array}$ & Stable disease & 5 \\
\hline GC_7 & Ramucirumab monotherapy & Progressive disease & 5 \\
\hline GC_8 & Two courses paclitaxel + ramucirumab & Progressive disease & 2 \\
\hline GC_9 & Paclitaxel + ramucirumab & Progressive disease & 9 \\
\hline GC_11 & Two courses: paclitaxel $\left(80 \mathrm{mg} / \mathrm{m}^{2}\right.$ days $\left.1,8,15\right)+$ ramucirumab $(8 \mathrm{mg} / \mathrm{kg}$ days 1,15$)$ & Progressive disease & 3 \\
\hline GC_12 & Ramucirumab (8 mg/kg every $14 \mathrm{~d}$ ) & Progressive disease & 6 \\
\hline GC_13 & Eight FOLFIRI + ramucirumab (8 mg/kg every $14 \mathrm{~d})$ & Stable disease & 8 \\
\hline GC_14 & Paclitaxel + ramucirumab & Progressive disease & 1 \\
\hline GC_15 & 11 injections: ramucirumab $8 \mathrm{mg} / \mathrm{kg}$ & Partial response & 8 \\
\hline GC_16 & 15 injections: ramucirumab $8 \mathrm{mg} / \mathrm{kg}$ & Stable disease & 7 \\
\hline GC_17 & 17 injections: ramucirumab $8 \mathrm{mg} / \mathrm{kg}+4$ injections paclitaxel & Stable disease & 11 \\
\hline GC_18 & 14 injections: ramucirumab $8 \mathrm{mg} / \mathrm{kg}$ & Stable disease & 8 \\
\hline GC_19 & Six injections: ramucirumab $8 \mathrm{mg} / \mathrm{kg}$ & Progressive disease & 5 \\
\hline
\end{tabular}

Chinese populations. It is also known as the poor-prognosis biomarker in endometrial carcinoma (Wang et al. 2015). In turn, mutations in the DNA repair gene TEX15 are known to be linked with a high risk of prostate and breast cancers (Lin et al. 2017).

We then calculated pathway activation levels of 3125 molecular pathways using the Oncobox software (Sorokin et al. 2018) and tested them for differential activation between the responder and nonresponder tumors (Supplemental Table S2). None of the pathways passed FDR threshold of 0.05 , but the most significantly differential pathway according to the Wilcoxon rank sum test was the "Nectin adhesion pathway (positive regulation of JNK cascade)." This pathway is a fragment of the Nectin adhesion regulatory network, which is responsible for downstream positive regulation of JNK (c-Jun amino-terminal kinase) cascade. This pathway appeared to be less active in the treatment responders compared with the nonresponders, mostly as a result of decreased expression of RAP1A, RAP1B, and $S R C$, which are upstream regulators of the CDC42 gene product (Fig. 2). Interestingly, activation of JNK signaling in GC cells can lead to enhanced resistance against platinum-based chemotherapeutics (Ye et al. 2015) and microtubule-targeting drugs including paclitaxel (Cui et al. 2017).

To assess the variance of the pathway activation level (PAL) and gene expression in the Nectin adhesion pathway (positive regulation of JNK cascade) between the cohorts of responders and nonresponders, we performed a nonpaired t-test and Wilcoxon test. We obtained for the $t$-test $P=0.01$, for the Wilcoxon test $P=0.0003$, and an absolute difference of average PAL scores of 0.35 , thus indicating that the observed difference is significant considering variances of PAL in each cohort. Similarly, we performed DESeq2 differential gene expression analysis for all genes forming this pathway (Table 4). For all individual genes, the expression differences did not reach a $P<0.05$ significance threshold, thus confirming previous findings on superior robustness of molecular pathways as cancer biomarkers (Borisov et al. 2014, 2017; Ozerov et al. 2016; Buzdin et al. 2018). 

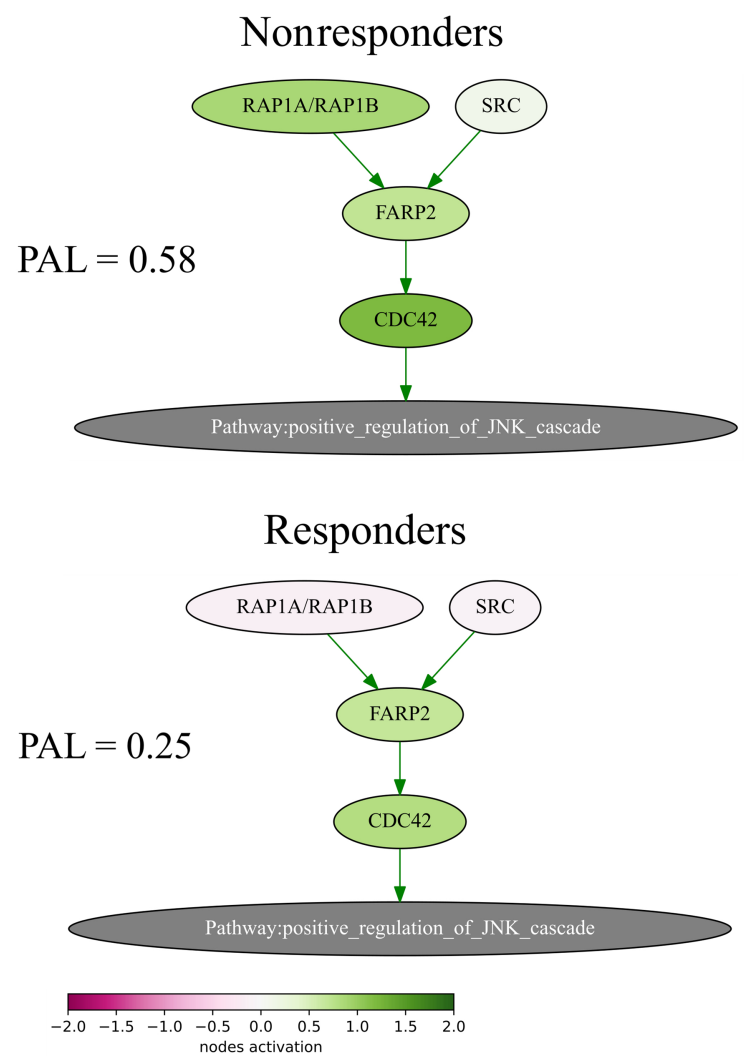

Figure 2. The nectin adhesion pathway (positive regulation of JNK cascade) was more active in the nonresponder tumors (upper panel) than in the responder tumors (lower panel). The pathway is shown as an interacting network in which green arrows indicate activation. Gene expression values were geometrically averaged for responders and nonresponders. The depth of color of each node of the network corresponds to the logarithms of the case-to-normal (CNR) expression rate for each node, in which "normal" is a geometric average between normal tissue samples. The normal tissue gene expression profiles for healthy donors were taken from the ANTE database (Suntsova et al. 2019). The scale represents the extent of up/down-regulation of the pathway nodes. PAL (pathway activation level) was calculated using the Oncobox software (described in Methods).

To our knowledge, none of those three differential genes (TEX15, CHRM3, LRFN1)/molecular pathways identified was previously connected with the sensitivity to ramucirumab in cancer cells.

We then used the Oncobox platform to build a personalized rating of targeted therapeutics for each individual GC sample according to their simulated abilities to inhibit aberrantly regulated molecular pathways and drug target genes. To this end, the RNA sequencing profiles obtained for the GC samples were individually compared with the set of profiles for normal stomach tissues obtained from the ANTE database (Suntsova et al. 2019). The output modeled drug efficiency values, termed balanced efficiency scores (BESs), which were obtained for 159 targeted therapeutics and prioritized drugs according to this score. A higher BES suggests higher predicted efficacy of a drug for an individual tumor (Poddubskaya et al. 2019b). We compared Oncobox BES values with the actual clinical outcomes of 15 ramucirumab-treated patients (Supplemental Table S3) and found that $B E S$ values could predict the $G C$ treatment response on ramucirumab-containing therapies with the quality metric area under the receiver operating characteristic (ROC) curve (ROC AUC) of 0.7 (Fig. 3A). 
Table 4. DESeq2 differential analysis results for the five genes of the "Nectin adhesion pathway (positive regulation of JNK cascade)" pathway

\begin{tabular}{lcc}
\hline Gene & $\log _{2}$ (fold change) & $P$-value \\
\hline RAP1A & -0.81 & 0.12 \\
CDC42 & -0.67 & 0.17 \\
RAP1B & -0.34 & 0.21 \\
SRC & -0.35 & 0.22 \\
FARP2 & -0.05 & 0.84 \\
\hline
\end{tabular}

A

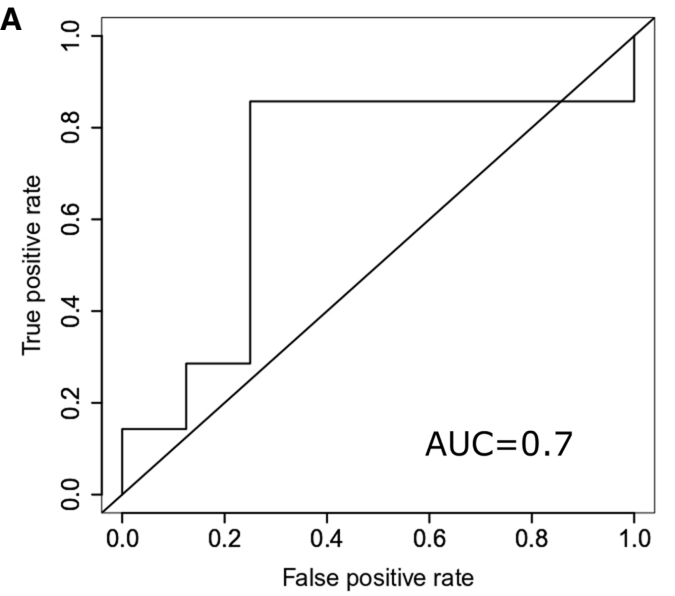

B

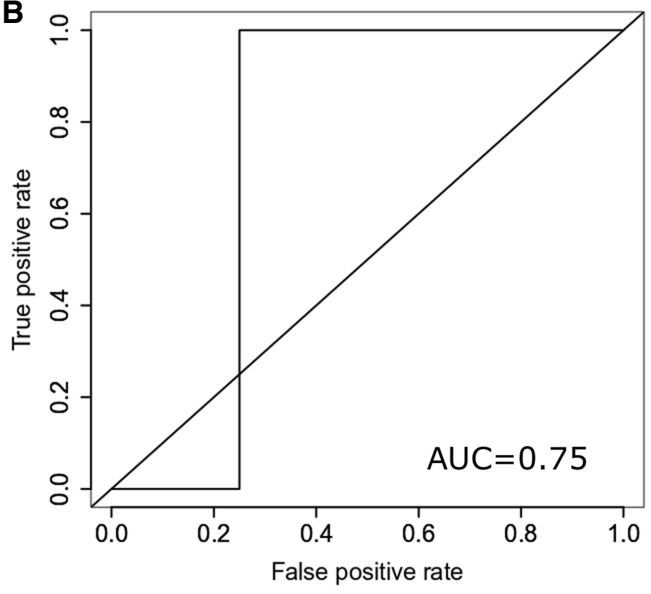

C

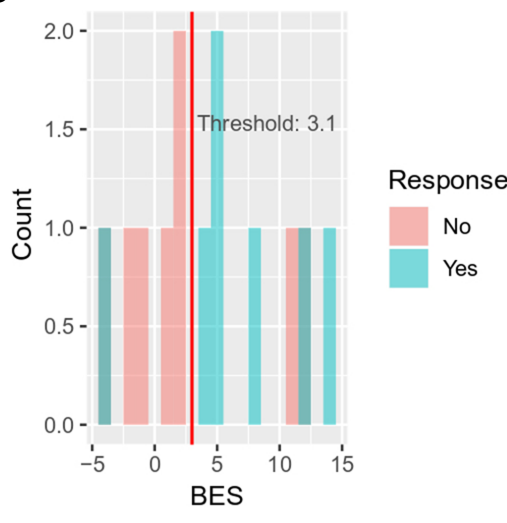

D

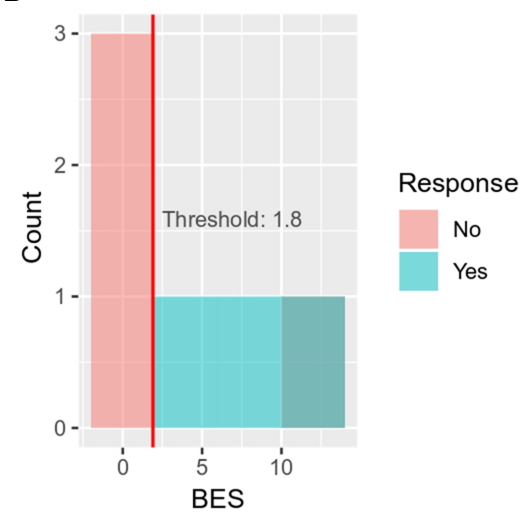

Figure 3. The receiver operating characteristic $(R O C)$ area under the curve (AUC) and histograms for predicting response to ramucirumab in gastric cancer (GC) patients using Oncobox balanced efficiency score (BES) values. (A) ROC AUC for the complete set of patients $(n=15)$. (B) ROC AUC for the patients who received ramucirumab monotherapy $(n=7)$. (C) Histogram and threshold value for the complete set of patients $(n=15)$. (D) Histogram and threshold value for the patients who received ramucirumab monotherapy $(n=7)$. The Oncobox BES of ramucirumab was used as a predictor of response. Patients were divided into two classes: "nonresponders," for progressive disease, and "responders," for stable disease and partial response (RECIST). Threshold values were assigned based on an assumption of the equality of type I and type II error rates. 


\begin{tabular}{lcc}
\hline $\begin{array}{l}\text { Table 5. Confusion matrix for a cohort of all patients (15 cases) using } \\
\text { the threshold } \mathrm{BES}=0.1 \text {, with sensitivity of } 0.62 \text { and specificity of } 0.14\end{array}$ \\
\hline $\begin{array}{c}\text { Cases predicted } \\
\text { as responders }\end{array}$ & $\begin{array}{c}\text { Cases predicted } \\
\text { as nonresponders }\end{array}$ \\
\hline Responders & 5 & 3 \\
Nonresponders & 6 & 1 \\
\hline
\end{tabular}

The AUC value is the universal characteristic of biomarker robustness depending on its sensitivity and specificity (Green and Swets 1966). This statistical approach is broadly applicable to different types of biomarkers in oncology (Borisov et al. 2014; Lezhnina et al. 2014; Liu et al. 2018; Tanioka et al. 2018; Zolotovskaia et al. 2018; Chen et al. 2019). It positively correlates with the quality of a biomarker and varies from 0.5 to 1 . The standard discrimination threshold is 0.7 , and the parameters with greater AUC are considered good-quality biomarkers, and vice versa (Boyd 1997).

The AUC value of 0.7 obtained for the whole set of patients (Fig. 3A) therefore suggests that the Oncobox BES value may serve as the good-quality predictive biomarker of GC clinical response for patients on ramucirumab. Importantly, reducing the patient cohort to those who received only ramucirumab monotherapy (seven cases) significantly improved performance of the BES-based predictions by increasing AUC to 0.75 (Fig. 3B). This can be explained by interference of other drugs (FOLFIRI, paclitaxel) in the non-monotherapy regimens.

To further study statistical performance of BES values, we pick up threshold values for cohorts of all patients ( 15 cases) and of those who received only ramucirumab monotherapy (seven cases). These thresholds were introduced to separate patients with different response status assuming equal importance for type I and type II errors (Fig. 3C,D); confusion matrices are shown in Tables 5 and 6 . The sensitivity and specificity values observed were, respectively, 0.83 and 0.71 for all patients, and 1 and 0.75 for the monotherapy cohort.

In an ongoing prospective trial of the Oncobox platform as a second-opinion tool for prescription of targeted therapeutics (NCT03724097), a BES threshold of 0.1 was arbitrarily set to discriminate between potentially effective and ineffective drugs. In this study, we tested this threshold for the original patient survival data. Eleven patients had greater and four patients had lower BES values (Fig. 4). Progression-free survival analysis revealed that Oncobox $\mathrm{BES}$ was able to predict better treatment outcomes for ramucirumab-containing therapies with hazard ratio $0.16(95 \% \mathrm{Cl}, 0.034-0.74, P=0.019$; Fig. 4) and log-rank test $P=0.013$. However, Cox proportional hazards analysis returned $\operatorname{Cox} P=0.31$. Such discrepant results between log-rank and Cox proportional hazards tests can be caused by small sample size. However, our further analysis showed that this BES threshold of 0.1 was suboptimal in terms of sensitivity and specificity values obtained, which were 0.62 and 0.14 , respectively (confusion matrix shown on Table 5). Our results, therefore, suggest that using a greater BES threshold can be beneficial for algorithmic discrimination of the potential treatment responders and nonresponders, at least for the case of using ramucirumab in GC. Here we

Table 6. Confusion matrix for a cohort of all patients (15 cases), with sensitivity of 0.83 and specificity of 0.71

\begin{tabular}{lcc}
\hline & $\begin{array}{c}\text { Cases predicted } \\
\text { as responders }\end{array}$ & $\begin{array}{c}\text { Cases predicted } \\
\text { as nonresponders }\end{array}$ \\
\hline Responders & 6 & 1 \\
Nonresponders & 2 & 5 \\
\hline
\end{tabular}


COLD SPRING HARBOR Molecular Case Studies
Ramucirumab response prediction in gastric cancer

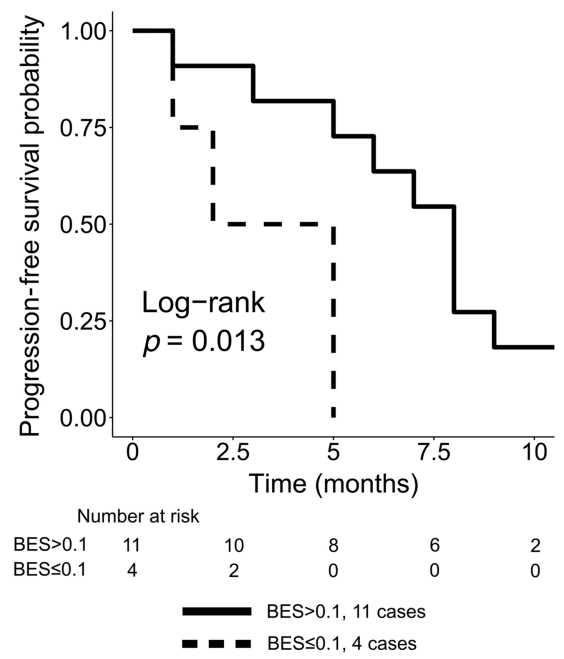

Figure 4. The Oncobox BES for ramucirumab predictive power for progression-free survival in GC patients receiving chemotherapy regimens with ramucirumab $(N=15)$. Cohorts of patients with BES values lower or higher than 0.1 are shown separately. The risk table is shown below the plot. Hazard ratio $=0.16(95 \% \mathrm{Cl}$, $0.034-0.74, P=0.019$ ). Log-rank test $P=0.013$.

exemplify that a threshold of 3.1 results in much better values of sensitivity and specificity for our group of samples (0.83 and 0.71 , respectively) (Table 6; Fig. 3C). Taken together, these data strongly suggest that the individual tumor responses for patients on ramucirumab can be predicted by using RNA sequencing profiles as the complex set of biomarkers.

\section{DISCUSSION}

Ramucirumab is the therapeutic monoclonal anti-VEGFR2 antibody that is approved for advanced GC or gastroesophageal junction adenocarcinoma either as monotherapy or in combination with paclitaxel (Fuchs et al. 2014; Wilke et al. 2014). It has been also approved for hepatocellular carcinoma (Zhu et al. 2019), non-small-cell lung cancer (Garon et al. 2014), and colorectal cancer (Tabernero et al. 2015). However, to our knowledge, so far there are no accepted biomarkers of ramucirumab efficiency in cancers (Bignucolo et al. 2017; Vlachostergios et al. 2018). We were also unable to find in the public domain any clinically annotated high-throughput gene expression profiles linked with the patient responses on treatment with ramucirumab. Here we for the first time experimentally analyzed RNA sequencing profiles of GC tumors with the available clinical outcomes of ramucirumab-containing treatment regimens.

When comparing treatment responders versus nonresponders, we found three statistically significantly differentially expressed genes: CHRM3, LRFN1, and TEX15. The most significantly differential molecular pathway was the "Nectin adhesion pathway (positive regulation of JNK cascade)." It is a fragment of the Nectin adhesion regulatory network that is responsible for positive regulation of the JNK cascade. We found that this pathway was more active in the nonresponders (Fig. 2) as a result of stronger expression of the RAP1A, RAP1B, and SRC genes, which encode upstream regulators of the CDC42 protein. CDC42 is a member of the Rho GTPase family that is involved in regulation of cell migration in various types of human cancers (Maldonado and Dharmawardhane 2018). In particular, CDC42 inhibition significantly decreased proliferation, migration, and invasion of GC cells 
(Du et al. 2016). Therefore, down-regulation of CDC42 activators, such as RAP1A, RAP1B, and SRC, may potentially be linked with tumor response to ramucirumab, because ramucirumab is an anti-VEGFR2 antibody that inhibits various signaling pathways including the SRC pathway (Clarke and Hurwitz 2013). Thus, decreased activation of the Nectin adhesion pathway (positive regulation of JNK cascade) in tumors may be linked to a response to ramucirumab in GC tissues.

Targeting molecular pathways abnormally activated in individual tumors was previously proposed to be a promising strategy for personalized selection of targeted therapies (Buzdin et al. 2018). Applicability of this approach to real-world cancer cases was tested in several clinical reports (Poddubskaya et al. 2018, 2019b). Here we present a retrospective cohort of advanced GC patients with known clinical outcomes for ramucirumab-containing therapies linked with high-throughput RNA sequencing profiles. Gene expression data were analyzed using the Oncobox bioinformatical platform for modeling the patient's tumor tissue sensitivity to ramucirumab. The predicted efficiencies were correlated with the actual tumor responses and progression-free survival data. Our results suggest that success of targeted therapies could be enhanced if guided by personalized analysis of gene expression. In this study, we experimentally profiled and bioinformatically investigated molecular data for $15 \mathrm{GC}$ patients, with the smallest predicted response group including only four patients (ramucirumab BES $<0.1$ ). As far as we know, this is currently the largest group of publicly available high-throughput gene expression profiles associated with known response statuses of patients being treated with ramucirumab. However, larger patient cohorts will be required to validate the trends observed here and to develop transcriptomics-based companion diagnostics for ramucirumab to personalize its use in GC.

\section{METHODS}

\section{Tissue Samples}

All experimental biosamples were FFPE tumor tissue blocks. All biosamples were evaluated by a pathologist to confirm the tumor tissue origin, and only the specimens with tumor cell content of $>50 \%$ were further investigated. Of them, five GC samples were obtained from M.F. Vladimirsky Moscow Regional Research Clinical Institute and 10 GC samples were obtained from N.N. Blokhin National Medical Research Center of Oncology. The patients were 10 men and five women, whose mean ages were 57.6 and $47.2 \mathrm{yr}$ old, respectively (with a range of 31-79 yr old).

The samples were clinically annotated and contained information about the patient's sex, age, diagnosis, and clinical history. For all the biosamples, informed written consent to participate in this study was collected from the patients or their legal representatives. The consent procedure and the design of the study were approved by the ethical committee of Vitamed Clinic, Moscow, protocol date 16.10.17. Clinical annotation of the tumor tissues investigated is summarized in Tables $1-3$.

\section{Preparation of Libraries and RNA Sequencing}

To isolate RNA, 10- $\mu \mathrm{M}$-thick paraffin slices were trimmed from each FFPE tissue block using a microtome. RNA was extracted from FFPE slices using QIAGEN RNeasy FFPE Kit following the manufacturer's protocol. RNA 6000 Nano or Qubit RNA Assay kits were used to measure RNA concentration. The RNA integrity number (RIN) was measured using the Agilent 2100 Bio-Analyzer. For depletion of ribosomal RNA and library construction, the KAPA RNA Hyper with rRNA erase kit (HMR only) was used. Different adaptors were used for multiplexing samples in one sequencing run. Library concentrations and quality were measured using the 
Qubit ds DNA HS Assay kit (Life Technologies) and Agilent TapeStation. RNA sequencing was done at the Department of Pathology and Laboratory Medicine, University of California Los Angeles, using Illumina HiSeq 3000 equipment for single-end sequencing, 50-bp read length, for $\sim 30$ million $(\mathrm{mln})$ raw reads per sample. A data quality check was done on Illumina SAV. Demultiplexing was performed with the Illumina Bcl2fastq2 v 2.17 program. Sequencing data were deposited in the NCBI SRA under accession ID PRJNA562149. Summary statistics of RNA sequencing (number of unmapped, uniquely mapped, and multimapped reads as well as average exon coverage) is depicted in Supplemental Table S1.

\section{Processing of RNA Sequencing Data}

RNA sequencing FASTQ files were processed with STAR aligner (Dobin et al. 2013) in "GeneCounts" mode with the Ensembl human transcriptome annotation (Build version GRCh38 and transcript annotation GRCh38.89). Ensembl gene IDs were converted to HGNC gene symbols using the Complete HGNC data set (https://www.genenames.org/; database version from 2017 July 13). Expression levels were established for 36,596 annotated genes with the corresponding HGNC identifiers. The minimum number of uniquely mapped reads was $3.75 \mathrm{mln}$ for studied biosamples, with the mean value of $9.78 \mathrm{mln}$. Differential gene expression analysis was performed using DESeq2 software (Love et al. 2014). PCA was performed for log-transformed DESeq2 normalized counts using the $R$ promp function.

\section{Molecular Pathway Analysis and Ranking of Target Drugs}

Pathway activation levels were established using the Oncobox analytic software (Sorokin et al. 2018) for 3125 molecular pathways extracted from the public databases Reactome (Croft et al. 2014), NCl Pathway Interaction Database (Schaefer et al. 2009), Kyoto Encyclopedia of Genes and Genomes (Kanehisa and Goto 2000), HumanCyc (Romero et al. 2005), Biocarta (Nishimura 2001), and QIAGEN pathway-central (available at https://www.qiagen.com/us/shop/genes-and-pathways/pathway-central/). The pathways investigated here are listed in Supplemental Table S1. The molecular pathways were visualized using the Oncobox pathway visualization/reconstruction tool (Sorokin et al. 2018; Buzdin et al. 2019a).

The PAL scores were calculated according to the Oncobox method (Borisov et al. 2020). For the pathways interrogated, gene expression values were geometrically averaged for the treatment responder and separately for the nonresponder biosamples. These averaged gene expression data were normalized on the normal tissue expression profiles extracted from the ANTE database (Suntsova et al. 2019). PAL scores were calculated as follows:

$$
\mathrm{PAL}=\frac{\sum_{i=1}^{n} \log _{10}\left(\frac{\text { average_gene_expression_cancer }}{\text { average_gene_expression_norm }}\right)}{n},
$$

in which PAL is pathway activation level, $n$ is number of genes in a pathway, average gene_expression_cancer is geometrically averaged gene expression value in all samples of a given cohort (treatment responders or nonresponders), and average_gene_expression_ norm is geometrically averaged normalized gene expression value in control samples. Ranking of target cancer drugs using BES was performed as described previously (Buzdin et al. 2018; Poddubskaya et al. 2019b; Tkachev et al. 2020). The Oncobox software returned a personalized list of target drugs in descending order of predicted efficacy. The observed clinical responses were used for validation of the Oncobox predictions using ROC AUC analysis. ROC AUC was calculated using the R ROCR package. Patient survival analysis was performed using the R ggsurvplot package. 
Competing Interest Statement

M.So., A.Ga., V.T., and A.B. have a financial relationship with Omicsway Corp. The remaining authors have nothing to disclose.

Received November 6, 2019; accepted in revised form February 3, 2020.

\section{ADDITIONAL INFORMATION}

\section{Data Deposition and Access}

The original sequencing data were deposited to NCBI SRA (https://www.ncbi.nlm.nih.gov/ sra) with accession number PRJNA562149, allowing free access.

\section{Ethics Statement}

For all the biosamples, informed written consent to participate in this study was collected from the patients or their legal representatives. The consent procedure and the design of the study were approved by the ethical committee of Vitamed Clinic, Moscow, protocol date 16.10.17.

\section{Acknowledgments}

We acknowledge the patients for their participation in this study.

\section{Author Contributions}

M.Se., E.P., M.So., A.Ga., and A.B. conceived of and designed the study. A.Gl., E.P., M.B., L.K., and E.M. collected and interpreted patient data. M.Se., M.Su., L.K., E.M., M.B., and E.P. isolated and prepared tissue samples. D.A. did histological analysis. M.Su. and D.A. performed molecular analyses. M.So., V.T., and A.B. analyzed the data. M.Se., A.B., and M.So. wrote the manuscript.

\section{Funding}

This study was supported by the OmicsWay research program in oncology, and by Russian Science Foundation grant 18-15-00061 (M.So., M.Su., and A.B.).

\section{REFERENCES}

Ajani JA, Ilson DH, Daugherty K, Pazdur R, Lynch PM, Kelsen DP. 1994. Activity of taxol in patients with squamous cell carcinoma and adenocarcinoma of the esophagus. J Natl Cancer Inst 86: 1086-1091. doi:10 $.1093 /$ jnci/86.14.1086

Al-Batran S-E, Hartmann JT, Probst S, Schmalenberg H, Hollerbach S, Hofheinz R, Rethwisch V, Seipelt G, Homann N, Wilhelm G, et al. 2008. Phase III trial in metastatic gastroesophageal adenocarcinoma with fluorouracil, leucovorin plus either oxaliplatin or cisplatin: a study of the Arbeitsgemeinschaft Internistische Onkologie. J Clin Oncol 26: 1435-1442. doi:10.1200/JCO.2007.13.9378

Albertsson M, Johansson B, Friesland S, Kadar L, Letocha H, Frykholm G, Wagenius G. 2007. Phase II studies on docetaxel alone every third week, or weekly in combination with gemcitabine in patients with primary locally advanced, metastatic, or recurrent esophageal cancer. Med Oncol 24: 407-412. doi:10.1007/ s12032-007-0028-6

Bang Y-J, Van Cutsem E, Feyereislova A, Chung HC, Shen L, Sawaki A, Lordick F, Ohtsu A, Omuro Y, Satoh T, et al. 2010. Trastuzumab in combination with chemotherapy versus chemotherapy alone for treatment of HER2-positive advanced gastric or gastro-oesophageal junction cancer (ToGA): a phase 3, open-label, randomised controlled trial. Lancet 376: 687-697. doi:10.1016/S0140-6736(10)61121-X

Belo A, Cheng K, Chahdi A, Shant J, Xie G, Khurana S, Raufman J-P. 2011. Muscarinic receptor agonists stimulate human colon cancer cell migration and invasion. Am J Physiol Gastrointest Liver Physiol 300: G749G760. doi:10.1152/ajpgi.00306.2010

Bignucolo A, De Mattia E, Cecchin E, Roncato R, Toffoli G. 2017. Pharmacogenomics of targeted agents for personalization of colorectal cancer treatment. Int J Mol Sci 18: E1522. doi:10.3390/ijms18071522

Borisov NM, Terekhanova NV, Aliper AM, Venkova LS, Smirnov PY, Roumiantsev S, Korzinkin MB, Zhavoronkov AA, Buzdin AA. 2014. Signaling pathways activation profiles make better markers of cancer than expression of individual genes. Oncotarget 5: 10198-10205. doi:10.18632/oncotarget.2548

Borisov N, Suntsova M, Sorokin M, Garazha A, Kovalchuk O, Aliper A, Initskaya E, Lezhnina K, Korzinkin M, Tkachev V, et al. 2017. Data aggregation at the level of molecular pathways improves stability of 
experimental transcriptomic and proteomic data. Cell Cycle 16: 1810-1823. doi:10.1080/15384101.2017 .1361068

Borisov N, Shabalina I, Tkachev V, Sorokin M, Garazha A, Pulin A, Eremin II, Buzdin A. 2019. Shambhala: a platform-agnostic data harmonizer for gene expression data. BMC Bioinformatics 20: 66. doi:10.1186/s12859019-2641-8

Borisov N, Sorokin M, Garazha A, Buzdin A. 2020. Quantitation of molecular pathway activation using RNA sequencing data. Methods Mol Biol 2063: 189-206. doi:10.1007/978-1-0716-0138-9_15

Boyd JC. 1997. Mathematical tools for demonstrating the clinical usefulness of biochemical markers. Scand J Clin Lab Invest Supp/ 227: 46-63. doi:10.1080/00365519709168308

Brar G, Shah MA. 2019. The role of pembrolizumab in the treatment of PD-L1 expressing gastric and gastroesophageal junction adenocarcinoma. Therap Adv Gastroenterol 12: 1756284819869767. doi:10.1177/ 1756284819869767

Buzdin AA, Zhavoronkov AA, Korzinkin MB, Roumiantsev SA, Aliper AM, Venkova LS, Smirnov PY, Borisov NM. 2014. The OncoFinder algorithm for minimizing the errors introduced by the high-throughput methods of transcriptome analysis. Front Mol Biosci 1: 8. doi:10.3389/fmolb.2014.00008

Buzdin A, Sorokin M, Garazha A, Sekacheva M, Kim E, Zhukov N, Wang Y, Li X, Kar S, Hartmann C, et al. 2018. Molecular pathway activation-new type of biomarkers for tumor morphology and personalized selection of target drugs. Semin Cancer Biol 53: 110-124. doi:10.1016/j.semcancer.2018.06.003

Buzdin A, Garazha A, Sorokin M, Glusker A, Aleshin A, Allina D, Suntsova M, Tkachev V, Borger P, Borisov N, et al. 2019a. RNA sequencing analysis for profiling activation of cancer-associated molecular pathways. $J$ Clin Oncol 37: e13032. doi:10.1200/JCO.2019.37.15_suppl.e13032

Buzdin A, Sorokin M, Garazha A, Glusker A, Aleshin A, Poddubskaya E, Sekacheva M, Kim E, Gaifullin N, Giese A, et al. 2019b. RNA sequencing for research and diagnostics in clinical oncology. Semin Cancer Biol 60: 311-323. doi:10.1016/j.semcancer.2019.07.010

Buzdin A, Sorokin M, Poddubskaya E, Borisov N. 2019c. High-throughput mutation data now complement transcriptomic profiling: advances in molecular pathway activation analysis approach in cancer biology. Cancer Inform 18: 1176935119838844. doi:10.1177/1176935119838844

Chen L, Zhou Y, Tang X, Yang C, Tian Y, Xie R, Chen T, Yang J, Jing M, Chen F, et al. 2019. EGFR mutation decreases FDG uptake in non-small cell lung cancer via the NOX4/ROS/GLUT1 axis. Int J Oncol 54: 370-380.

Clarke JM, Hurwitz HI. 2013. Targeted inhibition of VEGF receptor 2: an update on ramucirumab. Expert Opin Biol Ther 13: 1187-1196. doi:10.1517/14712598.2013.810717

Croft D, Mundo AF, Haw R, Milacic M, Weiser J, Wu G, Caudy M, Garapati P, Gillespie M, Kamdar MR, et al. 2014. The Reactome pathway knowledgebase. Nucleic Acids Res 42: D472-D477. doi:10.1093/nar/ gkt1102

Cui Y, Li Q, Li H, Wang Y, Wang H, Chen W, Zhang S, Cao J, Liu T. 2017. Asparaginyl endopeptidase improves the resistance of microtubule-targeting drugs in gastric cancer through IQGAP1 modulating the EGFR/ JNK/ERK signaling pathway. Onco Targets Ther 10: 627-643. doi:10.2147/OTT.S125579

Dobin A, Davis CA, Schlesinger F, Drenkow J, Zaleski C, Jha S, Batut P, Chaisson M, Gingeras TR. 2013. STAR: ultrafast universal RNA-seq aligner. Bioinformatics 29: 15-21. doi:10.1093/bioinformatics/bts635

Du D-S, Yang X-Z, Wang Q, Dai W-J, Kuai W-X, Liu Y-L, Chu D, Tang X-J. 2016. Effects of CDC42 on the proliferation and invasion of gastric cancer cells. Mol Med Rep 13: 550-554. doi:10.3892/mmr.2015 4523

Ferlay J, Shin H-R, Bray F, Forman D, Mathers C, Parkin DM. 2010. Estimates of worldwide burden of cancer in 2008: GLOBOCAN 2008. Int J Cancer 127: 2893-2917. doi:10.1002/ijc.25516

Fuchs CS, Tomasek J, Yong CJ, Dumitru F, Passalacqua R, Goswami C, Safran H, Dos Santos LV, Aprile G, Ferry DR, et al. 2014. Ramucirumab monotherapy for previously treated advanced gastric or gastro-oesophageal junction adenocarcinoma (REGARD): an international, randomised, multicentre, placebo-controlled, phase 3 trial. Lancet 383: 31-39. doi:10.1016/S0140-6736(13)61719-5

Fuchs CS, Shitara K, Di Bartolomeo M, Lonardi S, Al-Batran S-E, Van Cutsem E, Ilson DH, Alsina M, Chau I, Lacy $\mathrm{J}$, et al. 2019. Ramucirumab with cisplatin and fluoropyrimidine as first-line therapy in patients with metastatic gastric or junctional adenocarcinoma (RAINFALL): a double-blind, randomised, placebo-controlled, phase 3 trial. Lancet Oncol 20: 420-435. doi:10.1016/S1470-2045(18)30791-5

Garon EB, Ciuleanu T-E, Arrieta O, Prabhash K, Syrigos KN, Goksel T, Park K, Gorbunova V, Kowalyszyn RD, Pikiel J, et al. 2014. Ramucirumab plus docetaxel versus placebo plus docetaxel for second-line treatment of stage IV non-small-cell lung cancer after disease progression on platinum-based therapy (REVEL): a multicentre, double-blind, randomised phase 3 trial. Lancet 384: 665-673. doi:10.1016/S0140-6736(14) 60845-X

Green DM, Swets JA. 1966. Signal detection theory and psychophysics. Wiley, New York.

Kanehisa M, Goto S. 2000. KEGG: Kyoto Encyclopedia of Genes and Genomes. Nucleic Acids Res 28: 27-30. doi:10.1093/nar/28.1.27 
Kim S-H, Liu M, Jin HS, Park S. 2019. High genetic risk scores of ASIC2, MACROD2, CHRM3, and c2orf83 genetic variants associated with polycystic ovary syndrome impair insulin sensitivity and interact with energy intake in Korean women. Gynecol Obstet Invest 84: 225-236. doi:10.1159/000493131

Krupitskaya Y, Wakelee HA. 2009. Ramucirumab, a fully human mAb to the transmembrane signaling tyrosine kinase VEGFR-2 for the potential treatment of cancer. Curr Opin Investig Drugs 10: 597-605.

Lezhnina K, Kovalchuk O, Zhavoronkov AA, Korzinkin MB, Zabolotneva AA, Shegay PV, Sokov DG, Gaifullin NM, Rusakov IG, Aliper AM, et al. 2014. Novel robust biomarkers for human bladder cancer based on activation of intracellular signaling pathways. Oncotarget 5: 9022-9032. doi:10.18632/oncotarget.2493

Lin X, Chen Z, Gao P, Gao Z, Chen H, Qi J, Liu F, Ye D, Jiang H, Na R, et al. 2017. TEX15: a DNA repair gene associated with prostate cancer risk in Han Chinese. Prostate 77: 1271-1278. doi:10.1002/pros .23387

Liu T, Cheng G, Kang X, Xi Y, Zhu Y, Wang K, Sun C, Ye J, Li P, Yin H. 2018. Noninvasively evaluating the grading and IDH1 mutation status of diffuse gliomas by three-dimensional pseudo-continuous arterial spin labeling and diffusion-weighted imaging. Neuroradiology 60: 693-702. doi:10.1007/s00234-018-2021-5

Love MI, Huber W, Anders S. 2014. Moderated estimation of fold change and dispersion for RNA-seq data with DESeq2. Genome Biol 15: 550. doi:10.1186/s13059-014-0550-8

Maldonado MDM, Dharmawardhane S. 2018. Targeting Rac and Cdc42 GTPases in cancer. Cancer Res 78: 3101-3111.

Nishimura D. 2001. BioCarta. Biotech Softw Internet Rep 2: 117-120. doi:10.1089/152791601750294344

Ozerov IV, Lezhnina KV, Izumchenko E, Artemov AV, Medintsev S, Vanhaelen Q, Aliper A, Vijg J, Osipov AN, Labat I, et al. 2016. In silico pathway activation network decomposition analysis (iPANDA) as a method for biomarker development. Nat Commun 7: 13427. doi:10.1038/ncomms13427

Patel SP, Kurzrock R. 2015. PD-L1 Expression as a predictive biomarker in cancer immunotherapy. Mol Cancer Ther 14: 847-856. doi:10.1158/1535-7163.MCT-14-0983

Poddubskaya EV, Baranova MP, Allina DO, Smirnov PY, Albert EA, Kirilchev AP, Aleshin AA, Sekacheva MI, Suntsova MV. 2018. Personalized prescription of tyrosine kinase inhibitors in unresectable metastatic cholangiocarcinoma. Exp Hematol Oncol 7: 21. doi:10.1186/s40164-018-0113-x

Poddubskaya E, Buzdin A, Garazha A, Sorokin M, Glusker A, Aleshin A, Allina D, Moiseev A, Sekacheva M, Suntsova M, et al. 2019a. Oncobox, gene expression-based second opinion system for predicting response to treatment in advanced solid tumors. J Clin Oncol 37: e13143. doi:10.1200/JCO.2019.37 .15_suppl.e13143

Poddubskaya EV, Baranova MP, Allina DO, Sekacheva MI, Makovskaia LA, Kamashev DE, Suntsova MV, Barbara VS, Kochergina-Nikitskaya IN, Aleshin AA. 2019b. Personalized prescription of imatinib in recurrent granulosa cell tumor of the ovary: case report. Cold Spring Harb Mol Case Stud 5: a003434. doi:10 $.1101 /$ mcs.a003434

Rodon J, Soria J-C, Berger R, Miller WH, Rubin E, Kugel A, Tsimberidou A, Saintigny P, Ackerstein A, Braña I, et al. 2019. Genomic and transcriptomic profiling expands precision cancer medicine: the WINTHER trial. Nat Med 25: 751-758. doi:10.1038/s41591-019-0424-4

Romero P, Wagg J, Green ML, Kaiser D, Krummenacker M, Karp PD. 2005. Computational prediction of human metabolic pathways from the complete human genome. Genome Biol 6: R2. doi:10.1186/gb-2004-6$1-r 2$

Schaefer CF, Anthony K, Krupa S, Buchoff J, Day M, Hannay T, Buetow KH. 2009. PID: the pathway interaction database. Nucleic Acids Res 37: D674-D679. doi:10.1093/nar/gkn653

SEQC/MAQC-III Consortium. 2014. A comprehensive assessment of RNA-seq accuracy, reproducibility and information content by the Sequencing Quality Control Consortium. Nat Biotechnol 32: 903-914. doi:10.1038/nbt.2957

Smit EF, Garon EB, Reck M, Cappuzzo F, Bidoli P, Cohen RB, Gao L, O’Brien LM, Lee P, Zimmermann A, et al. 2018. Exposure-response relationship for ramucirumab from the randomized, double-blind, phase 3 REVEL trial (docetaxel versus docetaxel plus ramucirumab) in second-line treatment of metastatic nonsmall cell lung cancer. Cancer Chemother Pharmacol 82: 77-86. doi:10.1007/s00280-018-3560-5

Sorokin M, Kholodenko R, Suntsova M, Malakhova G, Garazha A, Kholodenko I, Poddubskaya E, Lantsov D, Stilidi I, Arhiri P, et al. 2018. Oncobox bioinformatical platform for selecting potentially effective combinations of target cancer drugs using high-throughput gene expression data. Cancers (Basel) 10: E365. doi:10 $.3390 /$ cancers 10100365

Suntsova M, Gaifullin N, Allina D, Reshetun A, Li X, Mendeleeva L, Surin V, Sergeeva A, Spirin P, Prassolov V, et al. 2019. Atlas of RNA sequencing profiles for normal human tissues. Sci Data 6: 36. doi:10.1038/s41597019-0043-4

Tabernero J, Cohn AL, Obermannova R, Bodoky G, Garcia-Carbonero R, Ciuleanu T-E, Portnoy DC, Van Cutsem E, Grothey A, Prausová J, et al. 2015. RAISE: a randomized, double-blind, multicenter phase III study of irinotecan, folinic acid, and 5-fluorouracil (FOLFIRI) plus ramucirumab (RAM) or placebo (PBO) in patients (pts) with metastatic colorectal carcinoma (CRC) progressive during or following first-line 
combination therapy with bevacizumab (bev), oxaliplatin (ox), and a fluoropyrimidine (fp). J Clin Orthod 33: 512.

Tanioka M, Fan C, Parker JS, Hoadley KA, Hu Z, Li Y, Hyslop TM, Pitcher BN, Soloway MG, Spears PA, et al. 2018. Integrated analysis of RNA and DNA from the phase III trial CALGB 40601 identifies predictors of response to trastuzumab-based neoadjuvant chemotherapy in HER2-positive breast cancer. Clin Cancer Res 24: 5292-5304. doi:10.1158/1078-0432.CCR-17-3431

Thuss-Patience PC, Kretzschmar A, Bichev D, Deist T, Hinke A, Breithaupt K, Dogan Y, Gebauer B, Schumacher G, Reichardt P. 2011. Survival advantage for irinotecan versus best supportive care as second-line chemotherapy in gastric cancer-a randomised phase III study of the Arbeitsgemeinschaft Internistische Onkologie (AIO). Eur J Cancer 47: 2306-2314. doi:10.1016/j.ejca.2011.06.002

Tkachev V, Sorokin M, Garazha A, Borisov N, Buzdin A. 2020. Oncobox method for scoring efficiencies of anticancer drugs based on gene expression data. Methods Mol Biol 2063: 235-255. doi:10.1007/978-1. 0716-0138-9_17

van de Vijver MJ, He YD, van't Veer LJ, Dai H, Hart AAM, Voskuil DW, Schreiber GJ, Peterse JL, Roberts C, Marton MJ, et al. 2002. A gene-expression signature as a predictor of survival in breast cancer. $N$ Engl J Med 347: 1999-2009. doi:10.1056/NEJMoa021967

Vlachostergios PJ, Lee A, Thomas C, Walsh R, Tagawa ST. 2018. A critical review on ramucirumab in the treatment of advanced urothelial cancer. Future Oncol 14: 1049-1061. doi:10.2217/fon-2017-0473

Wang Y, Li J, Wen S, Yang X, Zhang Y, Wang Z, Zhang Z. 2015. CHRM3 is a novel prognostic factor of poor prognosis in patients with endometrial carcinoma. Am J Transl Res 7: 902-911.

Wang C-T, Chen T-M, Mei C-T, Chang C-F, Liu L-L, Chiu K-H, Wu T-M, Lan Y-C, Liu W-S, Chen Y-H, et al. 2016. The functional haplotypes of modulate mRNA expression and associate with bladder cancer among a Chinese Han population in Kaohsiung city. Biomed Res Int 2016: 4052846.

Wilke H, Muro K, Van Cutsem E, Oh S-C, Bodoky G, Shimada Y, Hironaka S, Sugimoto N, Lipatov O, Kim T-Y, et al. 2014. Ramucirumab plus paclitaxel versus placebo plus paclitaxel in patients with previously treated advanced gastric or gastro-oesophageal junction adenocarcinoma (RAINBOW): a double-blind, randomised phase 3 trial. Lancet Oncol 15: 1224-1235. doi:10.1016/S1470-2045(14)70420-6

Yamaguchi K, Fujitani K, Nagashima F, Omuro Y, Machida N, Nishina T, Koue T, Tsujimoto M, Maeda K, Satoh T. 2018. Ramucirumab for the treatment of metastatic gastric or gastroesophageal junction adenocarcinoma following disease progression on first-line platinum- or fluoropyrimidine-containing combination therapy in Japanese patients: a phase 2, open-label study. Gastric Cancer 21: 1041-1049. doi:10.1007/ s10120-018-0811-4

Ye X-L, Zhao Y-R, Weng G-B, Chen Y-C, Wei X-N, Shao J-P, Ji H. 2015. IL-33-induced JNK pathway activation confers gastric cancer chemotherapy resistance. Oncol Rep 33: 2746-2752. doi:10.3892/or.2015.3898

Yoon HH, Bendell JC, Braiteh FS, Firdaus I, Philip PA, Cohn AL, Lewis N, Anderson DM, Arrowsmith E, Schwartz JD, et al. 2016. Ramucirumab combined with FOLFOX as front-line therapy for advanced esophageal, gastroesophageal junction, or gastric adenocarcinoma: a randomized, double-blind, multicenter Phase II trial. Ann Oncol 27: 2196-2203. doi:10.1093/annonc/mdw423

Zheng X-M, Zhang P, Liu M-H, Chen P, Zhang W-B. 2019. MicroRNA-30e inhibits adhesion, migration, invasion and cell cycle progression of prostate cancer cells via inhibition of the activation of the MAPK signaling pathway by downregulating CHRM3. Int J Oncol 54: 443-454.

Zhu AX, Kang Y-K, Yen C-J, Finn RS, Galle PR, Llovet JM, Assenat E, Brandi G, Pracht M, Lim HY, et al. 2019. Ramucirumab after sorafenib in patients with advanced hepatocellular carcinoma and increased $a$-fetoprotein concentrations (REACH-2): a randomised, double-blind, placebo-controlled, phase 3 trial. Lancet Oncol 20: 282-296. doi:10.1016/S1470-2045(18)30937-9

Zolotovskaia MA, Sorokin MI, Roumiantsev SA, Borisov NM, Buzdin AA. 2018. Pathway instability is an effective new mutation-based type of cancer biomarkers. Front Oncol 8: 658. doi:10.3389/fonc.2018.00658 


\section{COLD SPRING HARBOR Molecular Case Studies}

\section{RNA sequencing profiles and diagnostic signatures linked with response to ramucirumab in gastric cancer}

Maxim Sorokin, Elena Poddubskaya, Madina Baranova, et al.

Cold Spring Harb Mol Case Stud 2020, 6: a004945 originally published online February 14, 2020

Access the most recent version at doi: $10.1101 /$ mcs.a004945
Supplementary http://molecularcasestudies.cshlp.org/content/suppl/2020/02/18/mcs.a004945.D Material C1

References This article cites 63 articles, 5 of which can be accessed free at: http://molecularcasestudies.cshlp.org/content/6/2/a004945.full.html\#ref-list-1

License This article is distributed under the terms of the Creative Commons Attribution-NonCommercial License, which permits reuse and redistribution, except for commercial purposes, provided that the original author and source are credited.

Email Alerting Receive free email alerts when new articles cite this article - sign up in the box at the Service top right corner of the article or click here. 